\title{
Prevalence of malnutrition in hospital outpatients
}

\author{
S. Rust ${ }^{1}$, A. L. Cawood ${ }^{2}$, E. Walters ${ }^{1}$, R. J. Stratton ${ }^{2}$ and M. Elia ${ }^{3}$ \\ ${ }^{1}$ Nutrition and Dietetic Department, Southampton General Hospital, Tremona Road, Southampton SO16 6YD, UK, ${ }^{2}$ Medical \\ Affairs, Nutricia Ltd, White Horse Business Park, Trowbridge, BA14 OXQ, UK and ${ }^{3}$ Institute of Human Nutrition, University \\ of Southampton, Tremona Road, Southampton SO16 6YD, UK
}

\begin{abstract}
Malnutrition is an under-recognised and under-treated problem facing the $\mathrm{UK}^{(1)}$. It is recommended that all hospital outpatients are screened for malnutrition risk at their first clinic appointment ${ }^{(2)}$, despite this there remains little information on the prevalence of malnutrition in this population. The aim of this study was to establish prevalence of malnutrition in a range of outpatients using the 'Malnutrition Universal Screening Tool' ('MUST') (3) (http://www.bapen.org.uk).

Prospective malnutrition screening was carried out by a dietitian across two hospital sites in Southampton (Southampton General Hospital $(n=274)$ and Royal South Hants $(n=47))$ between July 2008 and June 2009. Screening for malnutrition was not routinely part of practice in outpatients across these sites. Three hundred and twenty one outpatients (mean age 54 (SD 16.7) years; $46 \%$ female) were screened for malnutrition using 'MUST', in a range of outpatient clinics including; gastroenterology (39\%); surgery (13\%), hepatology (17\%), oncology $(11 \%)$ and other $(20 \%)$. All patients could be weighed and screened using 'MUST'.

Overall prevalence of malnutrition using 'MUST' was $15.9 \%$ (95\% CI, 11.9-19.9\%): $10.9 \%$ medium risk; $5.0 \%$ high risk). There was a tendency for malnutrition risk to: increase with age ( $<65$ years versus $\geq 65$ years; $13.8 \%$ versus $20.8 \%, P=0.113$ ); be higher in women than men $(16.1 \%$ versus $15.7 \%, P=0.920)$, more common in the non-surgical than surgical clinics $(17.2 \%$ versus $7.1 \%$, $P=0.096)$ and more common in gastroenterology than non-gastroenterology clinics $(18.4 \%$ versus $14.3 \%, P=0.325)$ but none of the differences were significant. $5.6 \%$ of outpatients scored at step 1 (BMI score) and $12.1 \%$ scored at step 2 (weight loss score). The majority $(99.6 \%, n=276)$ of patients were happy to be screened.

This study shows that malnutrition is common (16\%) across outpatient clinics and patient compliance with 'MUST' screening is excellent. The absence of routine nutritional screening in these clinics meant that much malnutrition would have been unrecognised and untreated. Since the number of outpatient attendances outnumber hospital admissions several-fold, there is potential for identifying more malnutrition through screening in outpatients than inpatients.
\end{abstract}

1. Elia M. \& Russell C. (2009) Combating Malnutrition: Recommendations for Action. Report for the Advisory Board on Malnutrition, led by BAPEN.

2. NICE Guideline (2006). Nutrition Support in Adults. Clinical Guideline 32.

3. Elia M. (2003) The “'MUST"” Report. Nutritional Screening for Adults: A Multidisciplinary Responsibility. Redditch, Worcs.: BAPEN. 